\title{
Rotary oscillation control of a cylinder wake
}

\author{
By P. T. TOKUMARU AND P. E. DIMOTAKIS \\ Graduate Aeronautical Laboratories, California Institute of Technology 301-46, Pasadena, \\ CA 91125, USA
}

(Received 24 July 1989 and in revised form 19 July 1990)

Exploratory experiments have been performed on circular cylinders executing forced rotary oscillations in a steady uniform flow. Flow visualization and wake profile measurements at moderate Reynolds numbers have shown that a considerable amount of control can be exerted over the structure of the wake by such means. In particular, a large increase, or decrease, in the resulting displacement thickness, estimated cylinder drag, and associated mixing with the free stream can be achieved, depending on the frequency and amplitude of oscillation.

\section{Introduction}

It has long been appreciated that the dynamics of the flow over a bluff body are dominated by large-scale vortical structures shed in its wake. Weihs (1972) proposed a methodology for predicting the structure of such a wake and found that it could become wider, narrower, or remain the same, depending on the initial strength and spacing of these vortices. His work was primarily aimed at describing the wake behind an oscillating airfoil (e.g. Bratt 1953; Koochesfahani 1987). Nevertheless, Weihs's results do suggest the potential for effective control of wakes in general.

The effects of periodic forcing on a wake were investigated by Roberts (1985), and Roberts \& Roshko (1985). They demonstrated that a significant amount of control over the wake vortex structure and the associated mixing was possible, by pulsing one stream relative to the other. Okajima, Takata \& Asanuma (1975) examined the forces acting on a rotationally oscillating cylinder for Reynolds numbers, based on cylinder diameter, in the range

$$
40 \leqslant R e \equiv \frac{U_{\infty} d}{\nu} \leqslant 6 \times 10^{3},
$$

where $U_{\infty}$ is the free-stream velocity far ahead of the cylinder, $d$ is the cylinder diameter, and $v$ is the kinematic viscosity. His measurements were for a normalized peak rotation rate,

$$
\Omega_{1} \equiv \frac{v_{\theta_{1}}}{U_{\infty}}=\frac{d}{2 U_{\infty}} \dot{\theta}_{1}
$$

of 0.2 to 1.0 , and a forcing Strouhal number,

$$
S_{f} \equiv \frac{d}{U_{\infty}} f
$$

ranging from 0.05 to 0.3 , where $\dot{\theta}_{1}$ is the peak rotational rate of the cylinder, $v_{\theta_{1}}$ is the peak circumferential velocity, and $f$ is the forcing frequency. He noted a 
'synchronization' similar to that observed, for example, by Bishop \& Hassan (1964), Koopman (1967), and more recently, Ongoren \& Rockwell $(1988 a, b)$, and by Williamson \& Roshko (1988) for a cylinder in transverse and in-line oscillation. Both Okajima et al. and Bishop \& Hassan reported a hysteresis in their measurements for increasing and decreasing $S_{f}$. Investigations at comparable forcing frequencies, amplitudes and $R e$ were also performed by $W u$, Mo \& Vakili (1989). Taneda (1978) demonstrated that in the range $30 \leqslant R e \leqslant 300$, the 'dead water' region behind a cylinder can be removed for $\Omega_{1}$ sufficiently large $\left(\Omega_{1}>7\right.$ to 27 , depending on such factors as the cylinder span and tank width). For somewhat larger $\Omega_{1}$, the streamlines converged downstream. Similar behaviour was also documented by Williams \& Amato (1988) using a line of unsteady pulsing jets embedded in the trailing edge of the cylinder, at a comparable Reynolds number of $\mathbf{3 7 0}$.

In the work to be discussed here, we examined the efficacy of forced rotary oscillations of a (fixed axis) cylinder at a moderate Reynolds number of $1.5 \times 10^{4}$, for the purpose of controlling the unsteady separated flow in its wake. The effect of the cylinder oscillations on the structure of the wake was investigated using flow visualization, as well as surveys of the resulting wakes using laser-Doppler velocity measurements.

\section{Experimental facility}

The experiments documented here were performed in the $18 \mathrm{in}$. wide, by $19 \mathrm{in}$. deep, low speed water channel, as well as the $20 \times 20 \mathrm{in}$. free surface water channel at GALCIT. A 4 in. diameter Plexiglas cylinder was supported 10 in. above the bottom of the channel, using $0.5 \mathrm{in}$. thick Plexiglas fairings placed flush to the sidewalls of the channel. Drive belts and pulleys were entirely enclosed in the fairings and did not interfere with the flow. Rotation about the axis of the cylinder was achieved using a high-performance servo-controlled motor, capable of tracking an arbitrary command signal within the bandwidth and slewing rates that were investigated. This command signal was generated by a function generator, or a computer digital-to-analog converter output channel. Flow visualization was accomplished by introducing dye upstream of the cylinder, or through small holes on the cylinder surface at midspan. Streamwise mean and r.m.s. velocity profiles were measured using a Bragg cell frequency-shifted laser-Doppler velocimeter.

\section{Results and discussion}

The cylinder was rotated sinusoidally in time at a forcing frequency $f$ and a normalized peak rotational rate of $\Omega_{1}$ (see (2)), i.e.

$$
\frac{\dot{\theta} d}{2 U_{\infty}}=\Omega(t)=\Omega_{1} \sin (2 \pi f t) .
$$

$\Omega_{1}$ was chosen such that the peak circumferential velocity of the cylinder would be comparable with the velocity just outside the boundary layer of the cylinder (approximately two times the free-stream velocity). We anticipated that amplitudes of this magnitude or greater would be necessary to send the vorticity stored in the boundary layer into the wake in a regulated manner. For these experiments, we examined the control parameters $\Omega_{1}$ and $S_{f}$ in the range $0 \leqslant \Omega_{1} \leqslant 16$ and $0.17 \leqslant S_{f} \leqslant 3.3$. The free-stream velocity was approximately $15 \mathrm{~cm} / \mathrm{s}$, yielding a 


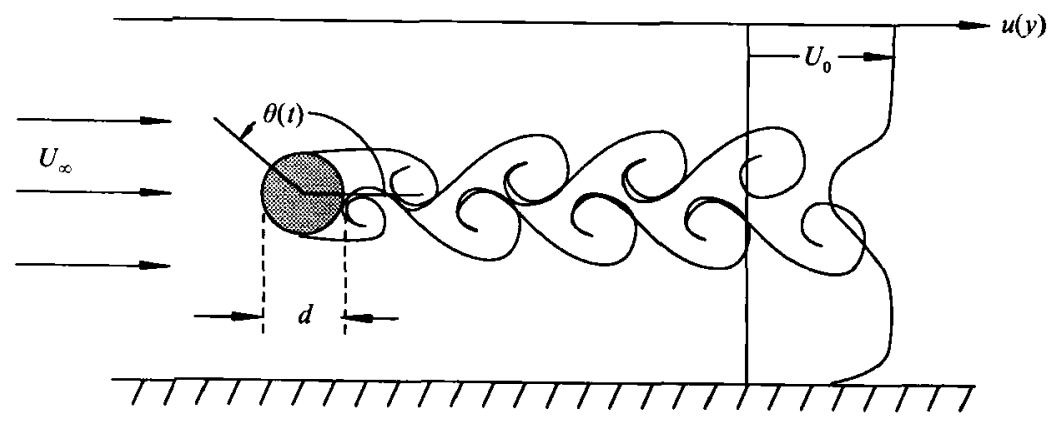

Fraure 1. Rotating cylinder flow geometry.

Reynolds number based on cylinder diameter of $R e=1.5 \times 10^{4}$. In addition, wake mean and r.m.s. velocity profiles were measured at a single streamwise station, located 4.5 diameters downstream of the cylinder axis. See figure 1.

\subsection{Displacement thickness and drag coefficient estimation}

In order to provide a quantitative measure of the effects of forcing on the flow over a cylinder and the resulting wake, the cylinder drag and wake displacement thickness were estimated from wake streamwise mean and r.m.s. velocity profiles. For flow that is two-dimensional, we can define the displacement thickness in terms of the integral

$$
\frac{\delta^{*}}{h} \equiv \int_{0}^{1}\left[1-\frac{u(\eta)}{U_{0}}\right] \mathrm{d} \eta .
$$

In this expression, $\eta \equiv y / h$ is the normalized vertical (cross-stream) position, $u(\eta)$ is the mean streamwise velocity, $y$ and $h$ are the vertical position and water channel depth respectively, and $U_{0}$ is the velocity in the (free-stream) region outside the cylinder wake and water channel boundary layers. See figure 1. By analogy, we may define the 'displaced area' for a three-dimensional flow, i.e.

$$
\frac{\Delta^{*}}{A} \equiv \int_{A}\left[1-\frac{u(\eta, \zeta)}{U_{0}}\right] \mathrm{d} \eta \mathrm{d} \zeta
$$

where $A$ is the test-section cross-sectional area, and $\zeta \equiv z / b$ is the normalized spanwise coordinate, with $z$ the spanwise coordinate, and $b$ the water channel span. Continuity then yields for $U_{\infty}$, the free-stream velocity far ahead of the cylinder,

$$
U_{\infty}=\int_{A} u(\eta, \zeta) \mathrm{d} \eta \mathrm{d} \zeta
$$

Using (6) and (7) we then find

$$
\frac{\Delta^{*}}{A}=1-\frac{U_{\infty}}{U_{0}}
$$

This expression is useful in that it provides information about the flow over an entire cross-section of the water channel, while requiring only the measurement of $U_{0}$ and $U_{\infty}$

In order to remove the contribution of the initial boundary layer (in the absence of the cylinder) to leading behaviour, it is useful to compare $U_{0}$ (with the cylinder in 


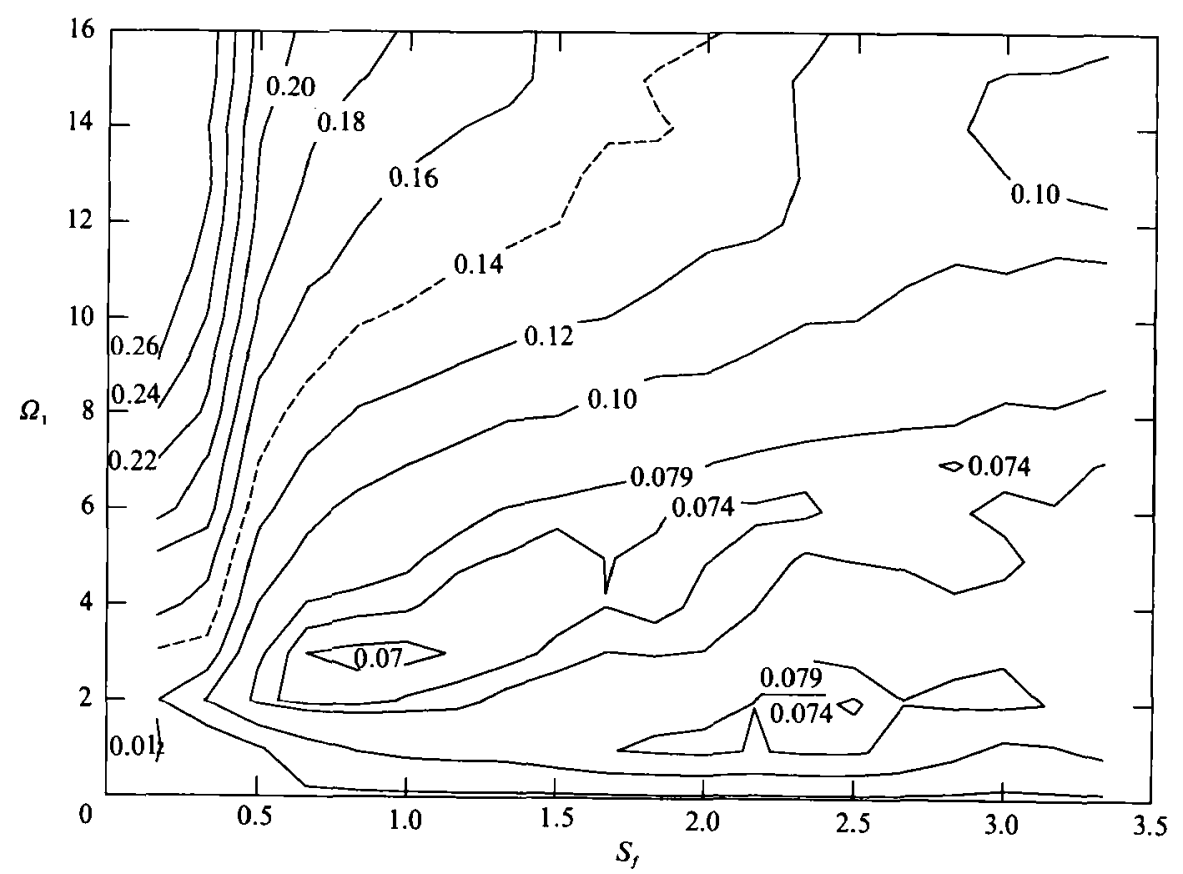

Fraure 2. Variation of $\hat{\Delta}^{*} / A$ with $S_{f}$ and $\Omega_{1}$.

place) with the undisturbed flow velocity in the absence of the cylinder $\hat{U}_{0}$, at the same streamwise location. This yields an expression for the approximate displaced area of the wake:

$$
\frac{\hat{A}^{*}}{A} \equiv 1-\frac{\hat{U}_{0}}{U_{0}}
$$

Figure 2 depicts the variation of $\hat{\Delta^{*}} / A$ with $S_{f}$ and $\Omega_{1}$. The dashed curve in this figure denotes the $\hat{\Delta}^{*} / A$ level for the unforced case. The parameter space above this curve represents an increase in $\hat{\Delta}^{*} / A$ over the unforced case, and below represents a decrease. Note that the displacement area can be made substantially larger or smaller by varying the frequency and amplitude of oscillation. The minimum for $\hat{A}^{*} / A$ over the range shown occurs in the neighbourhood of $S_{f} \approx 1$, and $\Omega_{1} \approx 3$, and is roughly half of the unforced case. Measurements for $\hat{A}^{*} / A$ were taken in the range $0.17 \leqslant S_{f} \leqslant 3.3$ in steps of about 0.17 , and $0 \leqslant \Omega_{1} \leqslant 16$ in steps of 1 . Each of the data points presented in figures 2 and 3 were averaged over 2 minutes (approximately 40 natural unforced shedding cycles). Wake midspan velocity profiles were measured for $S_{f}$ in the range of 0.15 to 1.4 , holding $\Omega_{1}$ fixed at 2 . Figure 3 compares the wake profiles for several $S_{f}$, and $\Omega_{1}=2$.

For finite test sections and flow that is two-dimensional in the mean, one can show that the sectional drag coefficient can be estimated by the expression (e.g. Dimotakis 1978):

$$
C_{D} \approx \frac{2 h / d}{\left(1-\delta^{*} / h\right)^{2}}\left[\int_{0}^{1} \frac{u}{U_{0}}\left(1-\frac{u}{U_{0}}\right) \mathrm{d} \eta-\int_{0}^{1} \frac{u^{\prime 2}-v^{\prime 2}}{U_{0}^{2}} \mathrm{~d} \eta+\frac{1}{2}\left(\frac{\delta^{*}}{h}\right)^{2}\right] .
$$

In this expression, $u^{\prime}$ and $v^{\prime}$ are the streamwise and cross-stream r.m.s., velocities and $\delta^{*}$ is the displacement thickness defined in (5). The displacement thickness and drag 


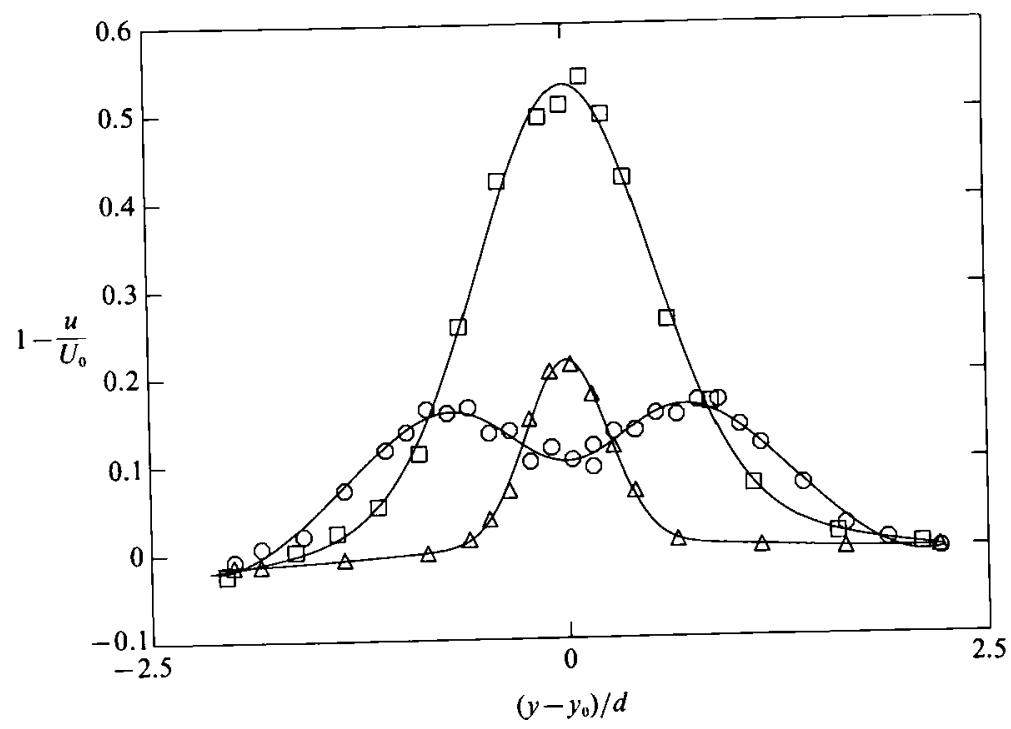

Figure 3. Cylinder wake mean velocity profiles, measured at midspan $\left(\Omega_{1}=2\right): \square$, unforced; $0, S_{f} \approx 0.2 ; \triangle, S_{f} \approx 1$.

coefficient estimates summarized in figure 4 were calculated from mean midspan velocity profiles, using (5) and (10), neglecting the fluctuating terms. As can be seen, there is a broad minimum in $C_{D}$ and $\delta^{*} / h$ around $S_{f}=1$. The estimated $C_{D}$ for the unforced case is a factor of six greater than for this forced case. Similarly, there is a factor of five reduction in $\delta^{*} / h$. Note that the value of $\delta^{*} / h$ for a particular $S_{f}$ and $\Omega_{1}$ is generally less than the corresponding value of $\hat{\Delta}^{*} / A$, indicating that there is an increase in $\delta^{*} / h$ outside the midspan region. Note also that since $C_{D}$ increases with $\delta^{*} / h$ it must also increase outside this midspan region. This spanwise variation in $\delta^{*} / h$ is due to the interaction of the sidewalls with the shed vortices. See Roberts (1985), Roberts \& Roshko (1985), Koochesfahani (1987), and Kurosaka et al. (1988). Another local minimum exists near $S_{f} \approx 0.2$. The flow visualization in figure 5 suggests that this corresponds to a mode in which the cylinder releases two vortices of opposite sign during each half-cycle. When one of these two vortices shed from the bottom appears on top, a dip is observed in the corresponding velocity profile. See the circles on figure 3 .

We should mention that Okajima et al. (1975) observed a hysteresis with increasing and decreasing $S_{f}$ and $\Omega_{1}=0.2$. We also observed some hysteresis for small values of $\Omega_{1}$ but none was discernible in experiments with $\Omega_{1} \geqslant 2$.

Finally, we suspect that had it not been for the finite height of the test section, the wake could have been made even larger by forcing at $S_{f}<0.2$.

\subsection{Flow visualization}

A clear picture of the control that can be exercised over the separated flow in the wake of the cylinder emerges from our flow visualization data. These demonstrate the overall narrowing or widening of the wake, depending on the values of $S_{f}$ and $\Omega_{1}$, the control parameters, as well as the formation of distinct vortical structures in the wake.

In figure 6 (plate 1), dye is introduced ahead of the cylinder by a comb of dye 

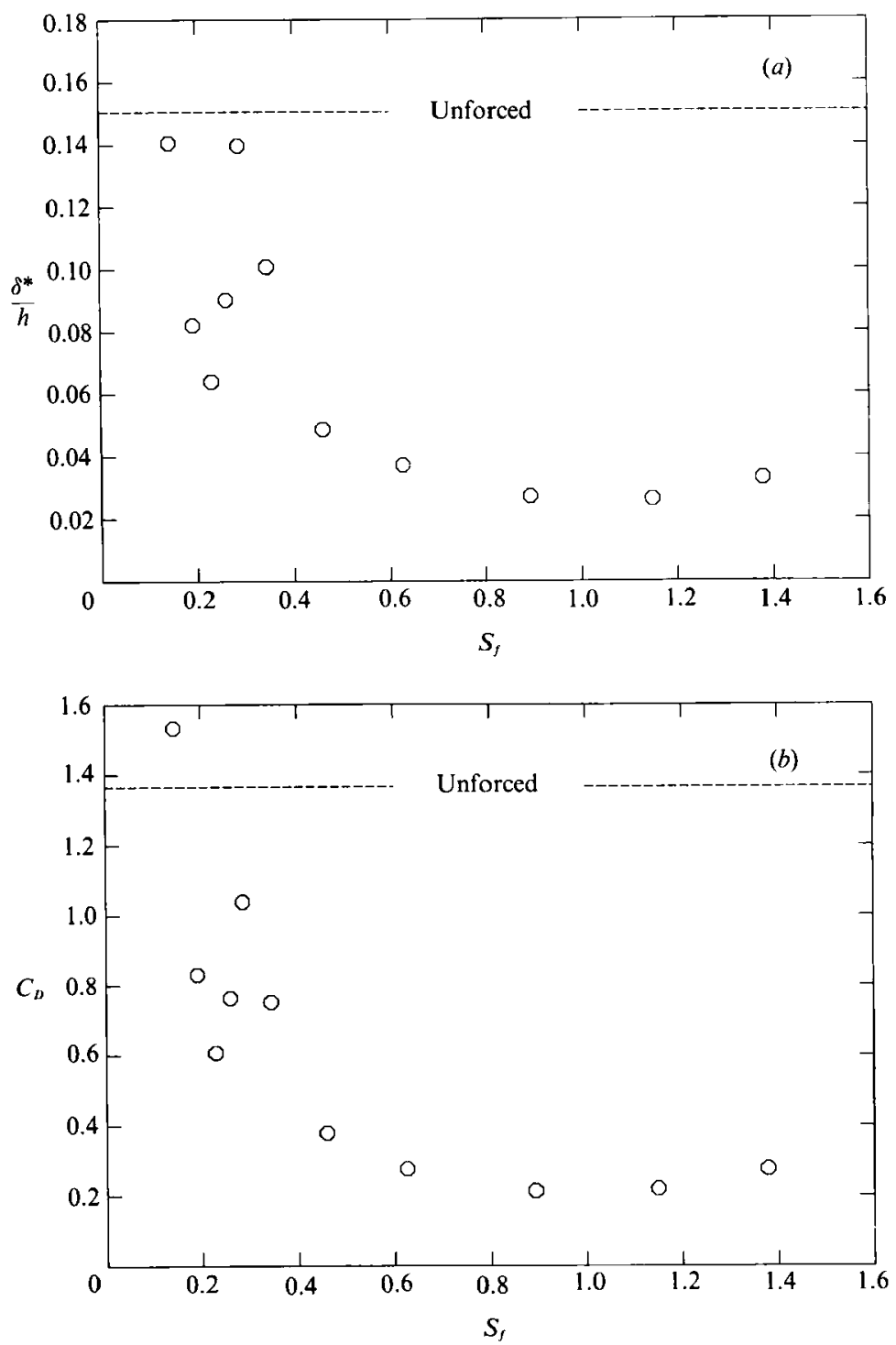

Figure 4. Cylinder wake displacement thickness and drag coefficient variation with $S_{f}$ $\left(\Omega_{1}=2\right):(a) \delta^{*} / h,(b) C_{D}$

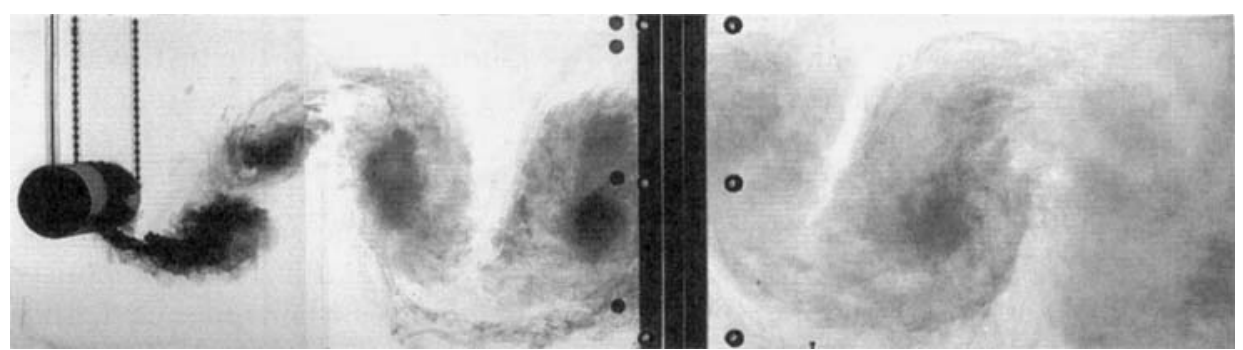

FIGURE 5. Forced shedding of two same-sign vortices during a half forcing cycle, $S_{f}=0.2, \Omega_{1}=2, R e=1.5 \times 10^{4}$. 
(a)

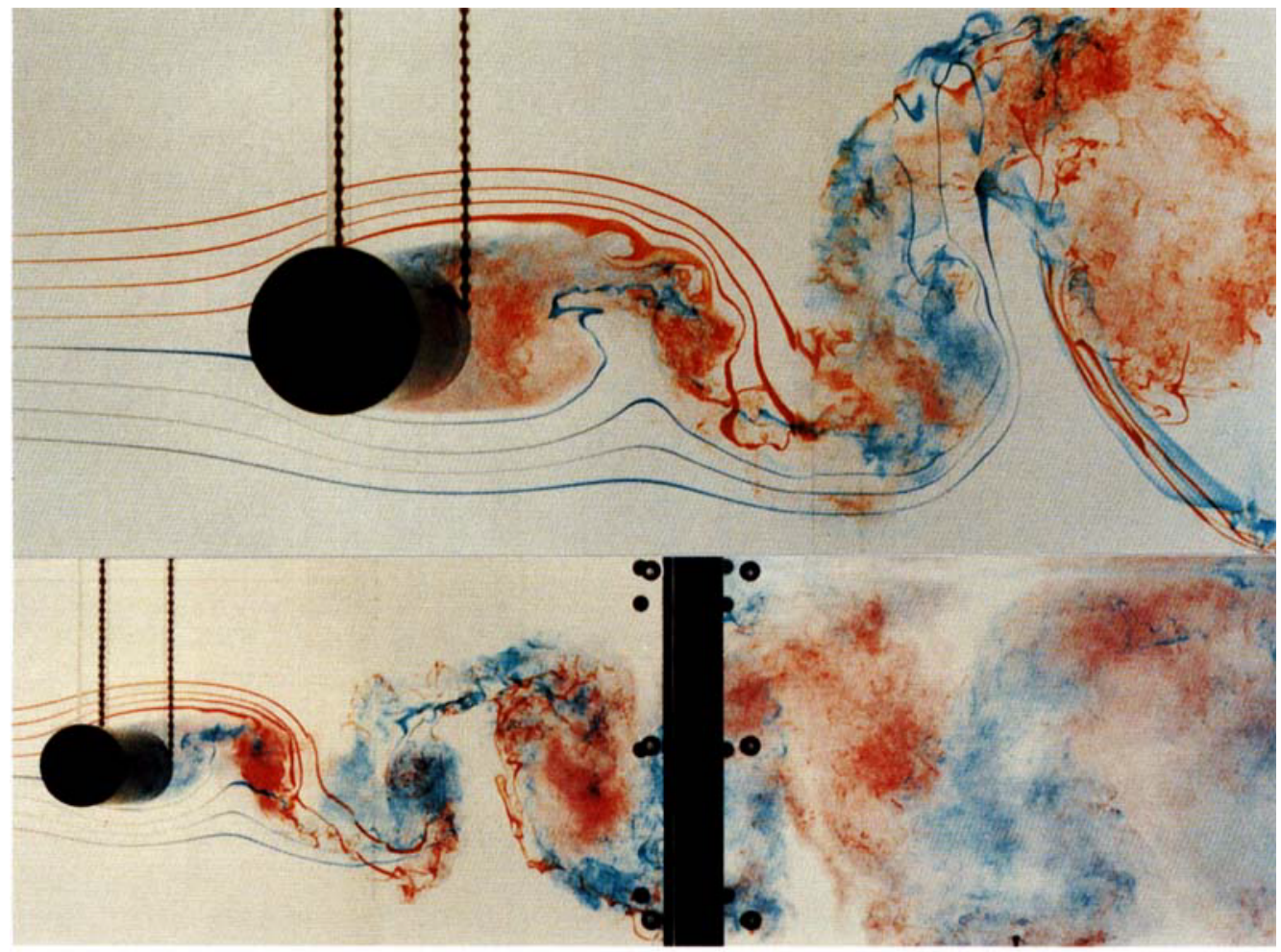

(b)

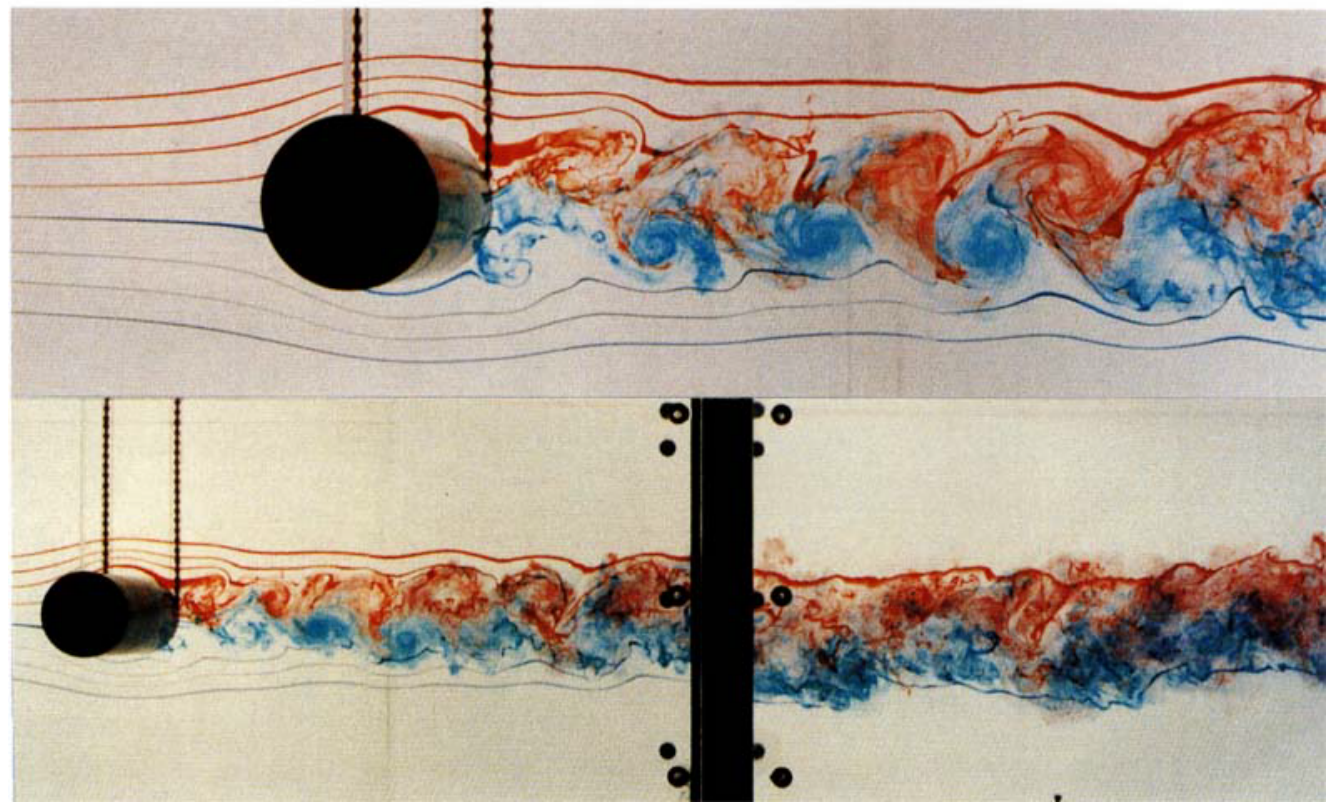

Figure 6. Close up and full flow visualization views with and without active control.

(a) Unforced (non-rotating) cylinder in uniform flow; (b) $S_{f} \sim 1, \Omega_{1} \approx 3$. 

(a)

(b)

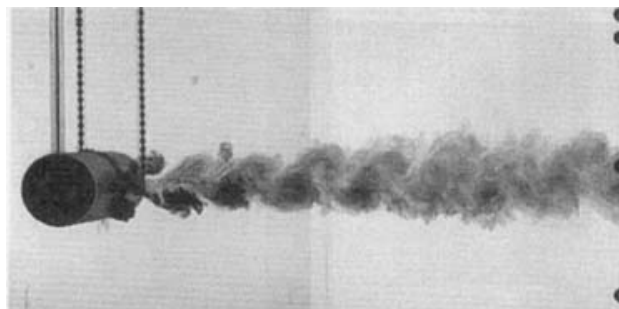

(c)

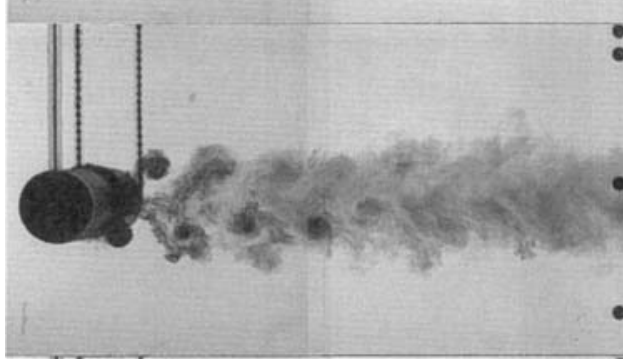

(d)

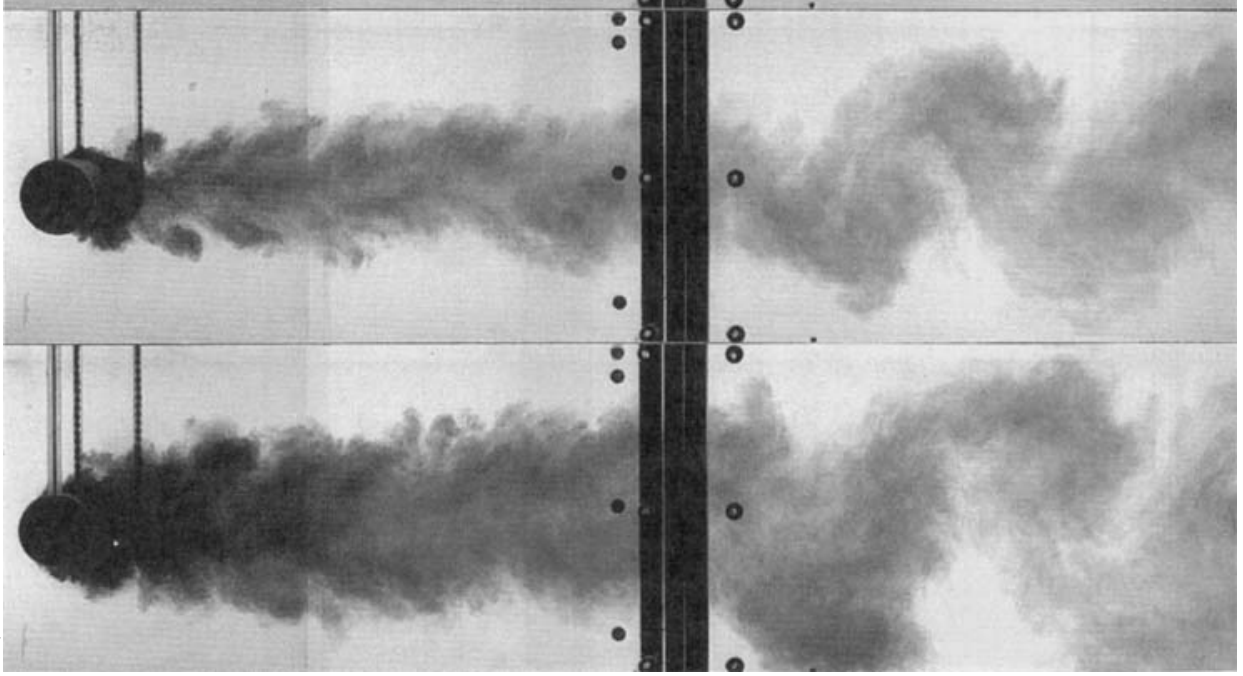

FiguRe 7. Forced shedding at fixed $S_{f}=1\left(R e=1.5 \times 10^{4}\right)$. (a) $\Omega_{1}=8$, (b) $\Omega_{1}=16$, (c) $\Omega_{1}=32$, (d) $\Omega_{1}=64$.

injectors. In the unforced case, figure $6(a)$, the dye can be seen to be dispersed and mixed across the full height of the test section, within a few diameters downstream of the cylinder. In contrast, the dye marker in the forced case corresponding to the minimum wake width found in $\$ 3.1$, figure $6(b)$, occupies approximately the same fraction of the test section height on leaving as it did upon entering.

The data in figures 7 and 8 (recorded with dye issuing from the surface of the cylinder) illustrate how the wake may be made wider or narrower by varying the control parameters $S_{f}$ and $\Omega_{1}$. Weihs (1972) examined this phenomenon by considering the mutual influence of the vortices in the wake and those being shed. Although his analysis is highly idealized, considering only point vortices in potential flow, it does provide an intuitive physical argument for the various trajectories taken by the vortices. Additional flow visualization data (also recorded with dye issuing from the surface of the cylinder), at fixed $\Omega_{1}=8$ and increasing $S_{f}$, are depicted in figures 9 and 10. 


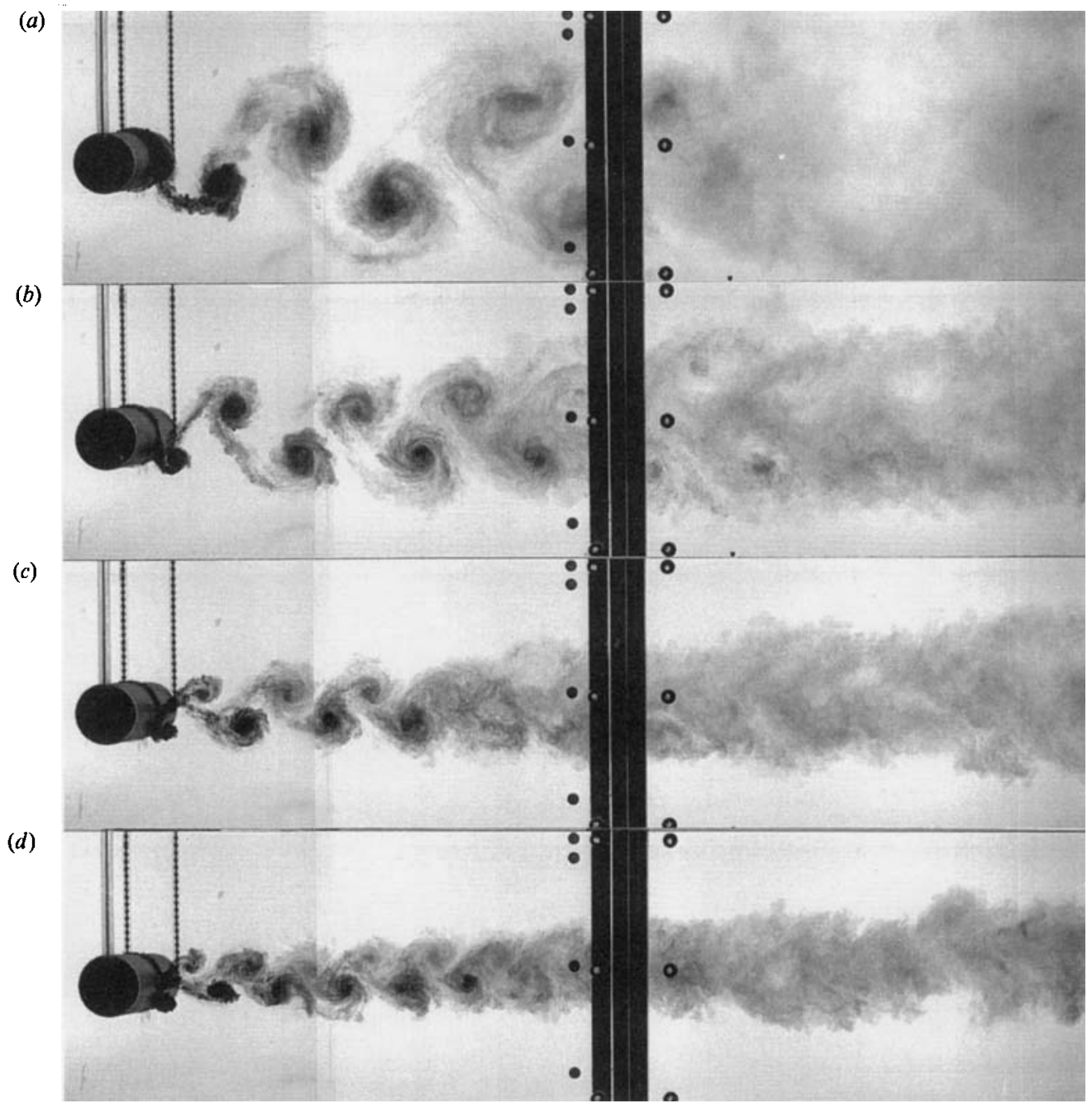

FiguRE 8. Mode II : wake structure is synchronized with the forcing frequency $\left(\Omega_{1}=8, R e=1.5 \times 10^{4}\right) .(a) S_{f}=0.3,(b) S_{f}=0.5,(c) S_{f}=0.7,(d) S_{f}=0.9$.

Several qualitatively different vortex shedding modes were observed. They are presented here as modes I to IV, in order of increasing frequency. In the forced mode I (e.g. figure 5), the cylinder releases two vortices of the same sign per half-cycle. This second vortex may be attributed to an additional separation caused by the most recently generated vortex. In mode II (e.g. figure 8), the wake structure is synchronized with the forced cylinder oscillation and persists beyond the end of the test section. In mode III (e.g. figure 9), the near-wake structure is also synchronized, but becomes unstable and evolves into a structure with a lower spatial frequency some distance downstream of the cylinder. In mode IV (e.g. figure 10), the effect of forcing is primarily observed in the shear layers separating from the cylinder. Note that while the flow visualization photographs of the forced cylinder wake, at the largest values of $S_{f}$ (cf. figure 10 , bottom), resemble those for the unforced case (cf. figure 11), we find that $\Delta^{*} / A$ in these forced cases is still noticeably less than in the unforced case. 
(a)

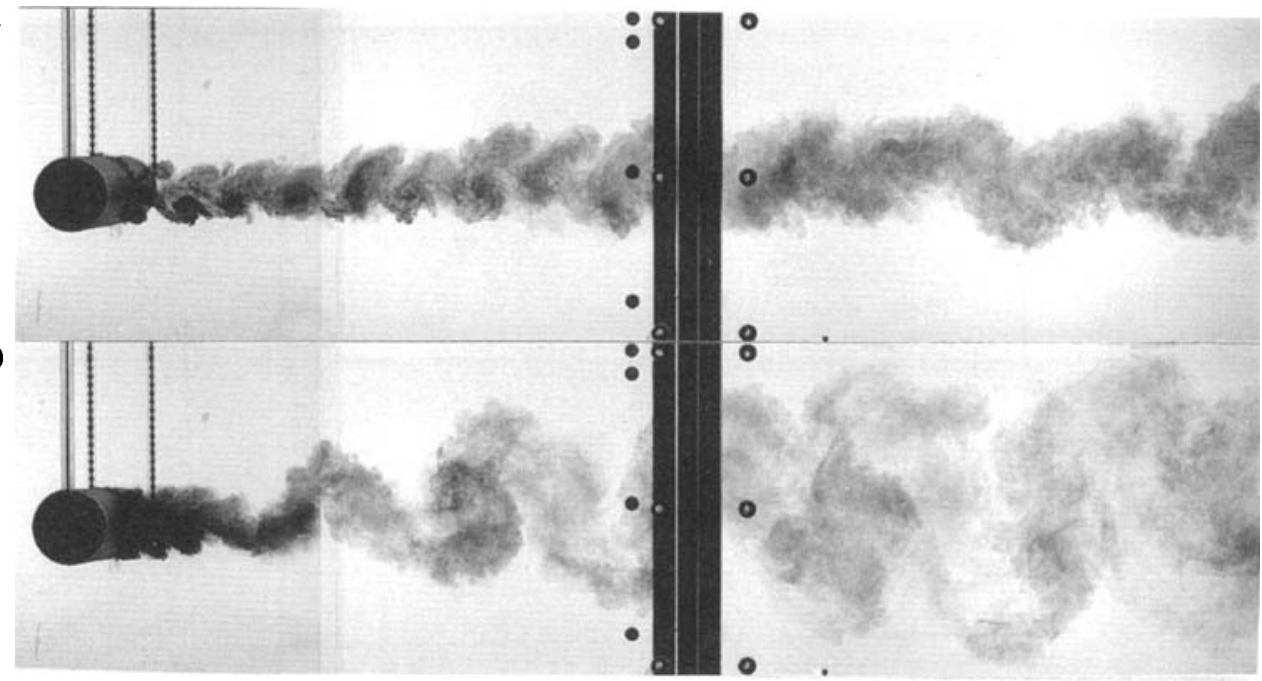

FIGURe 9. Mode III : transition from wake forcing to shear layer forcing $\left(\Omega_{1}=8\right.$, $\left.R e=1.5 \times 10^{4}\right) .(a) S_{f}=1.1,(b) S_{f}=1.5$.

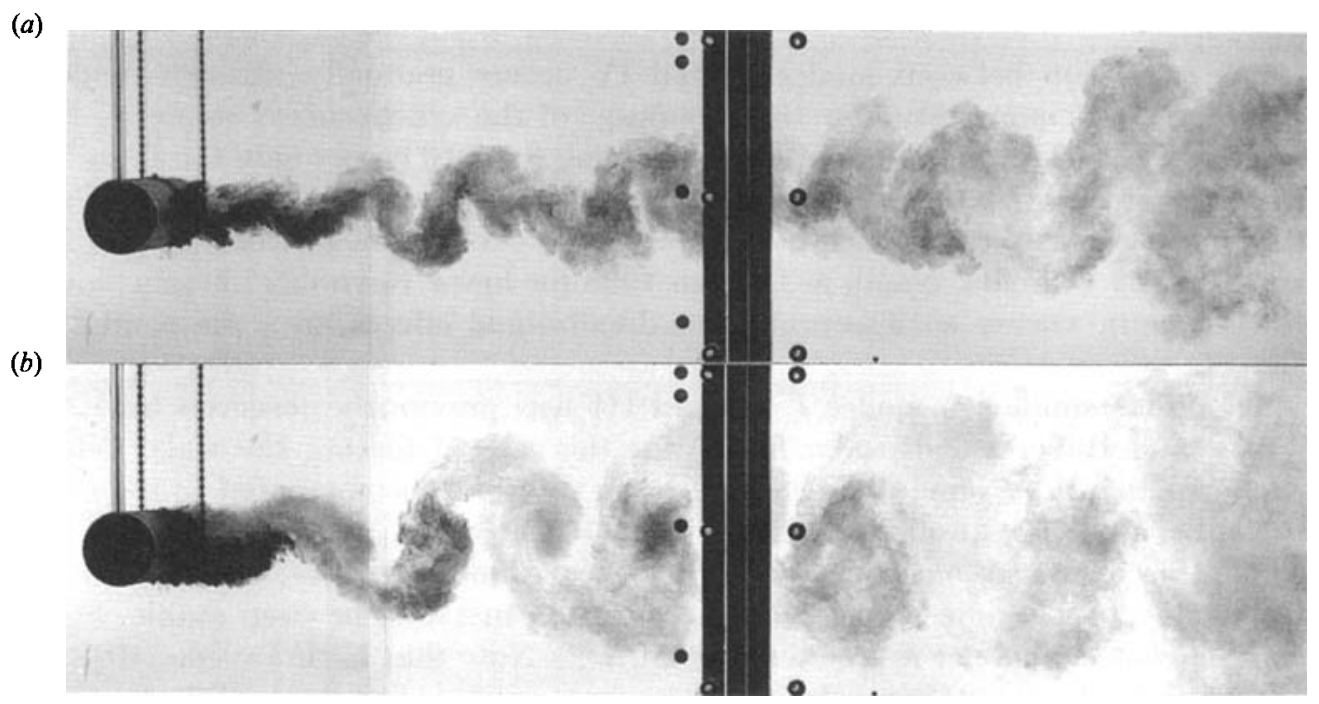

FIGURE 10. Mode IV : primarily shear layer forcing $\left(\Omega_{1}=8, R e=1.5 \times 10^{4}\right)$.

(a) $S_{f}=2.0,(b) S_{f}=3.3$.

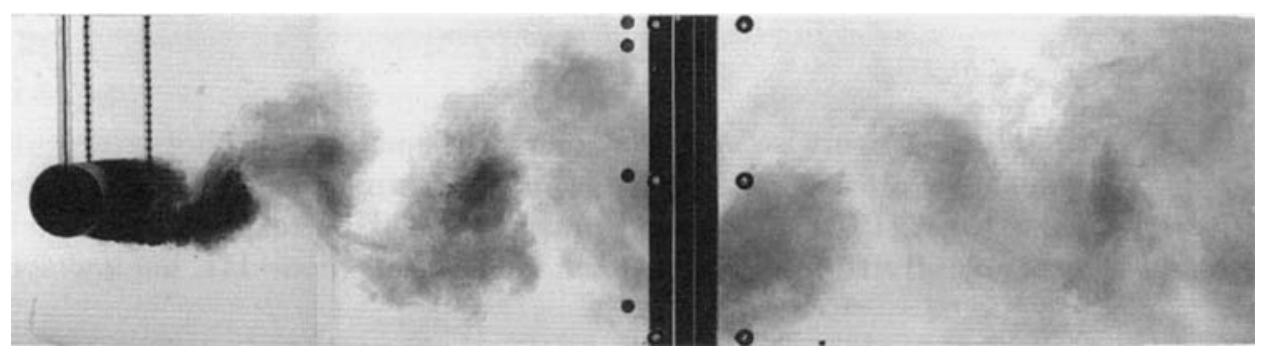

Figure 11. Natural (unforced) shedding. Flow visualized with dye issuing as for the data in figures 5, 7-10. 


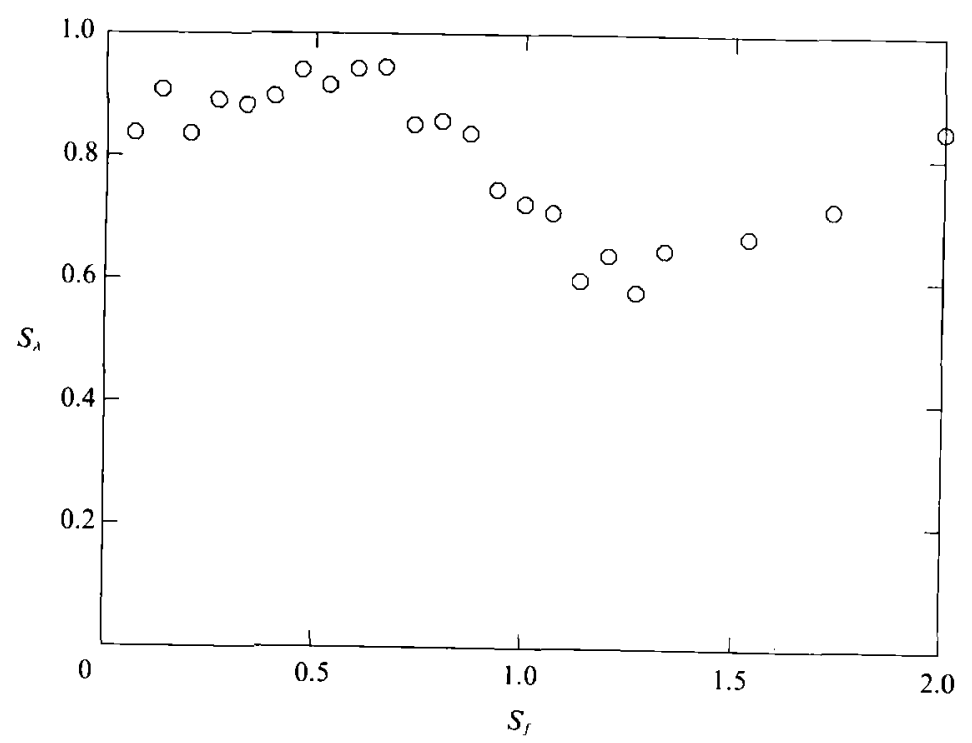

FIGURE 12. Variation of $S_{\lambda}$ with $S_{f}\left(\Omega_{1} \approx 8\right)$.

The transition between modes II and IV occurs gradually through mode III. Figures 8-10 demonstrate how the 'breakup' of the synchronized structure in this mode moves toward the cylinder with increasing $S_{f}$, until finally only the shear layers are forced. This breakup in the flow structure in modes III and IV may be attributable to the stability characteristics of the evolving mean velocity profiles (as suggested by Cimbala, Nagib \& Roshko 1988 for lower Reynolds number flows far downstream), vortex coalescence, three-dimensional effects, or some combination thereof.

Behaviour similar to modes I, II, and III was previously observed by Roberts (1985), and Roberts \& Roshko (1985) for the case of forcing the wake behind a splitter plate. More generally, the bluff-body wake can be compared to a nonlinear oscillator (e.g. Provansal, Mathis \& Boyer 1987) which, when forced, can reveal synchronized or phase-locked regions in their parameter spaces, as well as regions which may be periodic, quasi-periodic, chaotic, unstable, or even stable. See, for example, Guckenheimer \& Holmes (1983, Ch. 2). Note that both Taneda (1978), and Williams \& Amato (1988) show stable (non-vortex-shedding) regions in their frequency-amplitude parameter spaces.

Figure 12 shows the variation of the normalized phase speed,

$$
S_{\lambda} \equiv \frac{f}{U_{\infty}} \lambda
$$

with $S_{f} . \lambda$ is the initial streamwise vortex spacing, measured from photographs like those in figures 8-10. Note the rapid change in $S_{\lambda}$ around $S_{f}=1$. This change is reminiscent of Tritton's (1959) high-speed and low-speed vortex shedding modes. Our mode II resembles 'Tritton's high-speed mode and our mode III, his low-speed mode.

Observations of unforced vortex shedding show that vorticity is introduced into the flow from a more-or-less steady separation point, with the resulting vortical structures forming in the wake of the cylinder. This is in contrast to the forced 


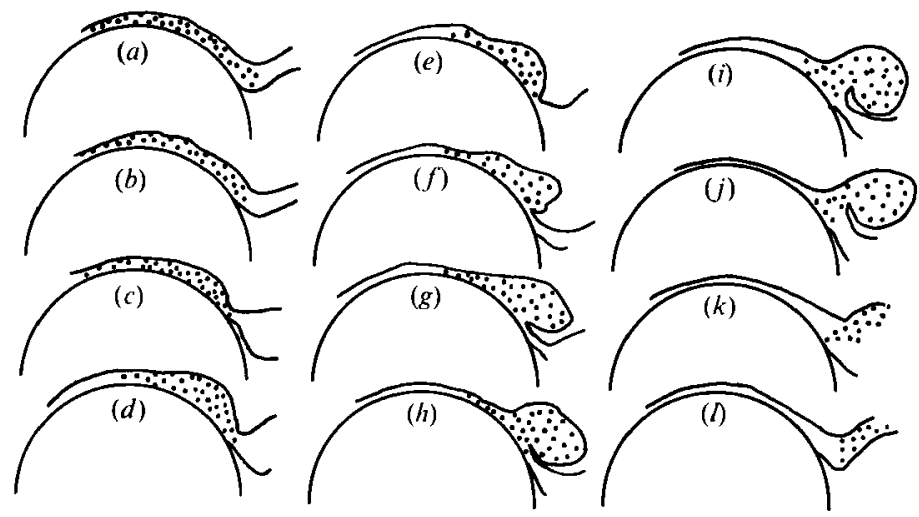

FIGURE 13. Near-wake region: forced shedding from the upper cylinder surface with $S_{f} \approx 1$ and $\Omega_{1} \approx 2$. The sequence $(a-l)$ represents one forcing period. The cylinder rotational velocity is zero in $(a)$ and $(l)$, clockwise in $(b-f)$, and counterclockwise in $(g-k)$ (dye was introduced ahead of the cylinder).

shedding process, representative of modes I-III, which is characterized by an unsteady separation 'point'. 'The forced shedding process for $S_{f} \approx 1$ and $\Omega_{1} \approx 2$ is illustrated in figure 13. The shaded areas in this figure denote fluid marked with dye introduced just ahead of the ten o'clock position on the cylinder. This forcing replaces the natural shedding mechanism with one that gathers vorticity close to the cylinder surface, and then releases it into the flow as a 'vortex'. It is plausible that this forcing mechanism will continue to operate for much higher Reynolds numbers, i.e. at least as high as the drag-critical Reynolds number, $R e \approx R e_{\text {cr }}>10^{5}$, and possibly higher.

The results reported here were recorded at moderate Reynolds numbers, i.e. $R e=$ $1.5 \times 10^{4}$. In an effort to assess Reynolds-number effects, some flow visualization and wake measurements were also performed on a cylinder whose surface was roughened by means of $0.017 \mathrm{in}$. diameter glass beads. This roughness had no discernible effect on the wake flow visualization or velocity measurements. In addition, some flow visualization was also performed on a smooth-surface cylinder, at a lower Reynolds number of $3.3 \times 10^{3}$. The resulting flows were found to be very similar. It may be interesting to note, however, that the effects at the higher forcing Strouhal numbers were somewhat less pronounced at this lower Reynolds number.

\subsection{A note about the interpretation of linear stability analyses}

There has been much interest in the use of linear stability analyses to deseribe unforced bluff body flows. See Koch (1985), Triantafyllou \& Chryssostomidis (1986), Monkewitz \& Nguyen (1987), Provansal et al. (1987), Chomaz, Huerre \& Redekopp (1988), Monkewitz (1988), and Karniadakis \& Triantafyllou (1989). Using lincar theory, it has been noted that the response to periodic forcing of the absolutely unstable near-wake region of a bluff body will be overwhelmed by the 'intrinsic' vortex shedding mode observed in the absence of external forcing. See Huerre \& Monkewitz (1985), Monkewitz \& Nguyen (1987), Chomaz et al. (1988), and Karniadakis \& Triantafyllou (1989). This argument is based on a linearized system and suggests that the periodic response will be overwhelmed because the transient response grows exponentially in time while the forcing is only periodic in time. Since the system cannot be considered linear for large excursions from the 'base' state, 
however, this argument can only be relied upon for infinitesimal forcing amplitudes and short times. It is not surprising perhaps, that this interpretation of the results of linear theory is not in accord with experiments and simulations which show that finite-amplitude forcing of the wake of a bluff body can result in a wake structure that is synchronous with the forcing, over a range of forcing frequencies and amplitudes, e.g. Koopman (1967), Provansal et al. (1957), and Karniadakis \& Triantafyllou (1989).

Provansal et al. (1987), examined the (nonlinear) Landau stability model (Landau 1944 ; Stuart 1958, 1960) and showed how it could be used to qualitatively describe the conditions necessary for synchronization at small but finite forcing amplitudes, and Reynolds numbers in the vicinity of the onset of vortex shedding. See also Landau \& Lifshitz (1987, §§26,30). Evidently, while the linear stability analysis, and hence the notion of the bluff-body wake as a globally unstable flow with a region of absolute instability, is useful in describing the system dynamics, given an a priori knowledge of the final averaged flow (Karniadakis \& Triantafyllou 1989), prediction of the final dynamic state, especially in the presence of finite external forcing requires a different approach.

\section{Conclusions}

In these experiments, we examined the efficacy of oscillatory cylinder rotation as an actuation mechanism for actively controlling the cylinder wake. We conclude that it is indeed possible to exercise considerable control over the cylinder wake via oscillatory rotary forcing. Not surprisingly, our experiments show that working in a control domain in which the structures shed are synchronous with the forcing provides the greatest control authority over the wake structure. In addition, while these results were obtained for moderate Reynolds numbers, i.e. $R e=1.5 \times 10^{4}$, preliminary evidence suggests that the description of the flow phenomena presented here is qualitatively the same over a large range of Reynolds numbers. The similarity of our results with those of others (e.g. Koopman 1967; Roberts 1985; Roberts \& Roshko 1985; Ongoren \& Rockwell 1988a,b; Williams \& Amato 1988, and Karniadakis \& Triantafyllou 1989), using various bluff bodies and forcing techniques, supports the proposition that the mechanisms by which the dynamies in the wake can be controlled are largely generic and have more to do with the ejection of circulation into the flow, rather than with the behaviour of the flow observed in the absence of forcing.

Finally, we note that the type of active programme control described in this paper relies on the regulated injection of circulation into the separated flow region, and the subsequent large-scale vortical interactions. This type of control which exploits the natural unsteadiness in the flow instead of reacting to it is in contrast to the feedforward control employed by Liepmann, Brown \& Nosenchuck (1982), and Liepmann \& Nosenchuck (1982), and the feedback control employed by Ffowcs-Williams \& Zhao (1989), for example. We believe that to control high-Reynolds-number, fully developed, separated turbulent flows, it may prove the more efficient and effective to programme the inevitable large-scale structures that dominate these flows, rather than to attempt to suppress them. The large reductions in the drag coefficient and the normalized wake displacement thickness that were achieved by the simple control scheme employed here supports this approach.

We would like to thank Hans Hornung, Tony Leonard, Richard Miake-Lye, Herb 
Gaebler, Harry Hamaguchi, and Pavel Svitek of GALCIT, as well as John Doyle of the Dept. of Electrical Engineering at Caltech, for their help and discussions. This research was sponsored by the Air Force Office of Scientific Research, URI AFOSR Grant No. F49620-86-C-0134.

\section{REFERENCES}

Bishop, R. E. D. \& Hassan, A. Y. 1964 The lift and drag on a circular eylinder oscillating in a flow field. Proc. R. Soc. Lond. A 277, 51-75.

Bratt, J. B. 1953 Flow patterns in the wake of a oscillating aerofoil. Aero. Res. Counc. R. \& M. 2773.

Chomaz, J. M., Huerre, P. \& Redekopp, L. G. 1988 Bifurcations to local and global modes in spacially developing flows. Phys. Rev. Lett. 60, 25-28.

Cimbala, J. M., Nagib, H. M. \& Roshro, A. 1988 Large structure in the far wakes of twodimensional bluff-bodies. J. Fluid Mech. 190, 265-298.

Dimotakis, P. E. 1978 Laser-Doppler velocimetry momentum defect measurements of cable drag at low to moderate Reynolds numbers. NCBC Rep. Contract N62583/77-M-R541.

Ffowes-Williams, J. E. \& Zhao, B. C. 1989 The active control of vortex shedding. J. Fluids Struct. 3, 115-122.

Guck enn eimer, J. \& Holmes, P. 1983 Nonlinear Oscillations, Dynamical Systems, and Bifurcations of Vector Fields. Springer.

Huerre, P. \& Monkewitz, P. A. 1985 Absolute and convective instabilities in free shear layers. J. Fluid Mech. 159, 151-168.

Karniadakis, G. E. \& Triantafyllou, G. S. 1989 Frequency selection and asymptotic states in laminar wakes. J. Fluid Mech. 199, 441-469.

Косн, W. 1985 Local instability characteristics and frequency determination of self-excited wake flows. J. Sound Vib. 99, 53-83.

Koochesfahani, M. M. 1987 Vortical pattern in the wake of an oscillating airfoil, 25th AIAA Aerospace Sciences Meeting, 12-15 January 1987, Reno, Nevada, AIAA Paper 87-0111.

Koochesfahani, M. M. \& Dimotakis, P. E. 1988 A cancellation experiment in a forced turbulent shear layer. In Proc. First Natl Fluid Dynamics Congr. 25-28 July 1988, Cincinatti, Ohio, vol. II, pp. 1204-1208; AIAA-88-3713-CP.

Koopman, G. H. 1967 The vortex wakes of vibrating cylinders at low Reynolds numbers. J. Fluid Mech. 28, 501-512.

Kurosaka, M., Christiansen, W. H., Goodman, J. R., Tirres, L. \& Wohlman, R. A. 1988 Crossflow transport induced by vortices. $A I A A J .26,1403-1405$.

Landa U, L. D. 1944 On the problem of turbulence. Dokl. Acad. Nauk. SSSR 44, 311-314.

Landau, L. D. \& Lifshitz, E. M. 1987 Fluid Mechanics, 2nd edn. Pergamon.

Liepmann, H. W., Brown, G. L. \& Nosenchuck, D. M. 1982 Control of laminar-instability waves using a new technique. J. Fluid Mech. 118, 187-200.

Limpmann, H. W. \& Nosenchuck, D. M. 1982 Active control of laminar-turbulent transition. $J$. Fluid Mech. 118, 201-204.

Monkewitz, P. A. 1988 The absolute and convective nature of instability in two-dimensional wakes at low Reynolds numbers. Phys. Fluids 31, 999-1006.

Monkewitz, P. A. \& Nauyen, L. N. 1987 Absolute instability in the near-wake of twodimensional bluff bodies. J. Fluids Struct. 1, 165-184.

Okajima, A., Takata, H. \& Asanuma, T. 1975 Viscous flow around a rotationally oscillating circular cylinder. Inst. Space \& Aero. Sci. (University of Tokyo), Rep. 532.

Ongoren, A. \& Rockwell, D. $1988 a$ Flow structure from an oscillating cylinder. Part 1. Mechanisms of phase shift and recovery in the near wake. J. Fluid Mech. 191, 197-223.

Ongoren, A. \& Rockwel.t, D. $1988 b$ Flow structure from an oscillating cylinder, Part 2. Mode competition in the near wake. J. Fluid Mech. 191, 225-245.

Provansal, M., Mathis, C. \& Boyer, L. 1987 Bénard-von Kármán instability: transient and forced regimes. J. Fluid Mech. 182, 1-22. 
RoBfrts, F. A. 1985 Effects of a periodic disturbance on structure and mixing in turbulent shear layers and wakes. Ph.D. thesis, California Institute of Technology.

Rовектs, F. A. \& Roshко. A. 1985 Effects of periodic forcing on mixing in turbulent shear layers and wakes. AIAA Shear Flow Control Conf. 12-14 March 1985, Boulder, CO, AIAA Paper 850570 .

Stcart. J. T. 1958 On the non-linear mechanics of hydrodynamic stability. J. Fluid Mech. 4. $1-21$.

Stuart, J. T. 1960 On the non-linear mechanies of wave disturbances in stable and unstable parallel flows. J. Fluid Mech. 9, 353-370.

TANEDA, S. 1978 Visual observations of the flow past a circular cylinder performing a rotatory oscillation. J. Phys. Soc. Japan 45, 1038-1043.

Triantafyllov. G. S., Triantafyliov, M. S. \& Chryssostomidis. C. 1986 On the formation of vortex streets behind stationary cylinders. J. Fluid Mech. 170, 461-477.

Trimron, D. J. 1959 Experiments on the flow past a circular cylinder at low Reynolds numbers. J. Fluid Mech. 6, 547-567.

WeIHs, D. 1972 Semi-infinite vortex trails, and their relation to oscillating airfoils. J. Fluid Mech. 54. $679-690$.

Williams, D. R. \& Aмато, C. W. 1988 Unsteady pulsing of cylinder wakes. In Proc. First Natl Fluid Dynamics Congr., 25-28 July 1988. Cincinatti. Ohio. pp. 731-737 (AIAA-88-3532-CP).

Willifamson, C. H. K. \& Roshro, A. 1988 Vortex formation in the wake of an oscillating cylinder. J. Fluids Struct. 2, 355-381.

Wu, J., Mo, J. \& VAKILI, A. 1989 On the wake of a cylinder with rotational oscillations. ALAA 2nd Shear Flow Conf., 13-16 March 1989, Tempe. AZ, AIAA Paper 89-1024. 